\title{
Expression and Purification of Natural N-Terminal Recombinant Bovine Pancreatic Trypsin Inhibitor from Pichia pastoris
}

\author{
Lili YAnG, ${ }^{a}$ Wen Dong, ${ }^{b}$ Jinchao He ${ }^{c}$ Xiubao Ren, ${ }^{a}$ and Weiqun YAN ${ }^{*, c}$ \\ ${ }^{a}$ Department of Immunology, Key Laboratory of Cancer Prevention and Therapy, Tianjin Medical University Cancer \\ Institute and Hospital; Tianjin 300060, P.R. China: ${ }^{b}$ Department of Orthopaedic Surgery, Tianjin Medical University First \\ Central Hospital; Tianjin 300192, P.R China: and ${ }^{c}$ Department of Biochemistry, College of Pharmacy, Jilin University; \\ Changchun 130021, P.R. China. Received December 27, 2007; accepted May 8, 2008; published online June 13, 2008
}

\begin{abstract}
Bovine pancreatic trypsin inhibitor (BPTI) is a natural non-specific serine protease inhibitor and possesses the ability to inhibit trypsin, chymotrypsin, plasmin and plasma kallikrein. The expression of BPTI in Escherichia coli and other systems has been reported. However, the preparation of recombinant BPTI (rBPTI) with correct N-terminus in Pichia pastoris has not been successful. A previous study showed that the preBPTI with the prepro leader sequence of alpha mating factor (AMF) was not processed into natural BPTI in P. pastoris. Now, we introduce a new method to prepare rBPTI, which carries a natural N-terminal amino acid residue, Arg-ProAsp, in $P$. pastoris using human serum albumin signal peptide corresponding to the pre sequence. The concentration of rBPTI in an 801 fermentor reached $900 \mathrm{mg} / \mathrm{l}$. We also explored a rapid and simple purification protocol for $\mathrm{rBPTI}$ and the purity of $\mathrm{rBPTI}$ reached $95-98 \%$ as evaluated by SDS-PAGE analysis. The sequencing results showed that the sequence of $\mathbf{N}$-terminal 15 amino acids of $r$ BPTI was consistent with that of natural BPTI. The inhibitory activity of rBPTI against trypsin was the same as natural BPTI and its $K_{\mathrm{i}}$ was $2.6 \pm 0.1 \times 10^{-9}$. The therapeutic effect of rBPTI on acute pancreatitis was identified in rats.
\end{abstract}

Key words bovine pancreatic trypsin inhibitor; human serum albumin signal peptide; Pichia pastris; fermentation; purification

Bovine pancreatic trypsin inhibitor (BPTI, trade name: aprotinin) is a member of the Kunitz-type inhibitor family and possesses the ability to inhibit trypsin, chymotrypsin, plasmin and plasma kallikrein. ${ }^{1,2}$ It is a single chain alkaline polypeptide consisting of 58 amino acid residues crosslinked by three pairs of disulphide bonds. ${ }^{3,4)}$ Aprotinin is mainly used for long-term treatment of acute pancreatitis. Since the 1990s, it has been utilized in surgery to protect blood platelets and decrease haemorrhage and capillary haemorrhage, and in the treatment of coagulation disorders. ${ }^{5,6)}$ Aprotinin has become one of the most important biochemical drugs.

However, the current aprotinin for commercial use has been extracted from bovine organs such as lungs, pancreas etc. It is difficult to control the purity because of the complex technical procedures, and mad cow disease has had a serious influence on the material source of aprotinin. These drawbacks above have limited the aprotinin application in clinical practice. On the other hand, with the continuous new pharmacodynamics discoveries about aprotinin, its clinical application has continued to spread and market demand has ceaselessly increased. Therefore, the preparation of recombinant BPTI (rBPTI) using genetic engineering technology is the only way to solve the above problems.

It has been reported that rBPTI can be expressed in Escherichia coli, ${ }^{7-9)}$ Saccharomyces cerevisiae, ${ }^{10,11)}$ Pichia pastoris $^{12,13)}$ and other expression systems, ${ }^{14)}$ but there are some insurmountable disadvantages such as low yield, incorrect folding and cleavage.

Especially in $P$. pastoris, rBPTI with a correct $\mathrm{N}$-terminal "Arg-Pro-Asp" cannot be obtained with the use of a prepro leader sequence of alpha mating factor (AMF), because the processing protease Kex 2 is not able to correctly cleave the pro sequence of AMF to produce mature BPTI with natural $\mathrm{N}$-terminal amino acid residues as true in human mutant proalbumin. It is still an unsolved problem how to establish an expression system of rBPTI which is consistent with natural BPTI both in structure (correct N-terminal and spatial conformation) and in biological activities, as well as how to obtain a stable and high yield suitable for industrialization.

Based on our research on P. pastoris and human serum albumin (HSA), we studied the rBPTI preparation from many aspects including signal peptide, promoter, yeast strain and yeast preferred codons. Finally, an expression system of $P$. pastoris suitable for rBPTI industrialization was established with the HSA signal peptide. It was confirmed that rBPTI possessed a natural structure and biological activities.

The purpose of this paper is to describe the expression of rBPTI induced by HSA signal peptide in $P$. pastoris and to explore the methods of large-scale fermentation and purification.

\section{MATERIALS AND METHODS}

Construction of Plasmid PICZC/HSA-BPTI Two synthetic oligonucleotides encoding the HSA signal peptide: forward primer 5'-CGAAACGATGAAGTGGGTAACCTTTATTTCCCTTCTTTTTCTCTTTAGCTCGGCTTAC- ${ }^{\prime}{ }^{\prime}$ and reverse primer 5'-TCGAGTAAGCCGAGCTAAAGAGAAAAAGAAGGGAAATAAAGGTTACCCACTTCATCGTTT$3^{\prime}$ containing $B s t \mathrm{BI}$ and $X h o \mathrm{I}$ (underline) were annealed at $100^{\circ} \mathrm{C}$. Then the DNA fragment of HSA signal peptide was subcloned to pPICZaC (Invitrogen, Carlsbad, CA, U.S.A.) and was digested with $B s t \mathrm{BI}$ and $X h o \mathrm{I}$ to generate plasmid PICZC/HSA.

To clone BPTI gene, reverse transcription polymerase chain reaction (RT-PCR) was done with primers (forward primer, 5'-CAAAGCCTCGAGACCTGACTTCTGCCTA-3'; reverse primer $5^{\prime}$-GGTTCTTCTAGAGCCCTTAAGCACC$\left.3^{\prime}\right)$ and the total RNA extracted from bovine lung as the template. Following digestion by $X h o \mathrm{I}$ and $X b a \mathrm{I}$, the PCR fragment was subcloned to pPICZC/HSA and treated with the 
same endonucleases to generate recombinant enkaryotic expression plasmid PICZC/HSA-BPTI. The recombinant plasmid was identified by restriction analysis and nucleotide sequencing.

Transformation of $\boldsymbol{P}$ pastoris Yeast transformation was performed according to the manufacturer's instruction. The recombinant plasmid DNA was linearized with PmeI, and then was transformed into $P$. pastoris X-33, KM71 and SMD1168 (Invitrogen, Carlsbad, CA, U.S.A.) The yeast strains transformed with pPICZaC plasmid and without any treatment served as a negative control. ${ }^{15)}$ The cells were plated on yeast extract peptone dextrose (YPD) plates $(1 \%$ yeast extract, $2 \%$ peptone and $2 \%$ dextrose) containing Zeocin (Invitrogen, Carlsbad, CA, U.S.A.), of which the concentrations were $100 \mu \mathrm{g} / \mathrm{ml}, 250 \mu \mathrm{g} / \mathrm{ml}, 500 \mu \mathrm{g} / \mathrm{ml}$ and $1000 \mu \mathrm{g} / \mathrm{ml}$ and incubated at $28^{\circ} \mathrm{C}$, respectively.

Expression and Optimization of rBPTI Initial screening of transformants was carried out prior to large-scale expression. After transformants were incubated for $72 \mathrm{~h}, 20$ positive clones from each of the strains of X-33, KM71 and SMD1168 were randomly selected, inoculated in $10 \mathrm{ml}$ buffered glycerol-complex (BMGY) medium in a test tube ( $1 \%$ yeast extract, $2 \%$ peptone, $1.34 \%$ yeast nitrogen base, $100 \mathrm{~mm}$ potassium phosphate, $4 \times 10^{-5} \%$ biotin and $1 \%$ glycerol) and agitated at $28{ }^{\circ} \mathrm{C}$ for $48 \mathrm{~h}$. The DNAs of transformants were extracted and then amplified with primers of BPTI cloning described above. The presence of the BPTI gene in transformants was confirmed by gene sequencing of PCR products. The DNAs of non-transformed yeast and pPICZaC-transformed yeast were extracted as control. The positive transformants identified by PCR were expressed in $10 \mathrm{ml}$ buffered methanol-complex (BMMY) medium in a test tube $(1 \%$ yeast extract, $2 \%$ peptone, $1.34 \%$ yeast nitrogen base, $100 \mathrm{~mm}$ potassium phosphate, $4 \times 10^{-5 \%}$ biotin and $0.5 \%$ methanol). Pure methanol was added every $24 \mathrm{~h}$ to a final concentration of $0.5 \%$. After $96 \mathrm{~h}$ induction, the transformants culture broth was centrifuged and the supernatant was collected. The supernatant was analyzed using SDSPAGE and trypsin inhibition assay. A clone of the high expression level was inoculated in $10 \mathrm{ml}$ of BMGY/BMMY medium with different $\mathrm{pH}$ of $3.0,3.5,4.0,4.5,5.0,5.5,6.0$, $6.5,7.0$ to determine the optimal $\mathrm{pH}$. In order to determine the optimal temperature, the selected clone was inoculated in $21 \mathrm{BMGY} / \mathrm{BMMY}$ medium at different temperatures of $24^{\circ} \mathrm{C}, 26^{\circ} \mathrm{C}, 28^{\circ} \mathrm{C}$ and $30^{\circ} \mathrm{C}$ for $96 \mathrm{~h}$, respectively.

Fermentation of rBPTI After the optimal $\mathrm{pH}$ and temperature were determined, rBPTI was further fermented for large-scale production in an 801 fermentor (Bioflo. 5000 New Brunswick Scientific Inc., Edison, NJ, U.S.A.). The protocol for fermentation including the fermentation parameters and phases was performed according to the Invitrogen Manual for $P$. pastoris fermentation. The fermentor was sterilized with 251 of basal salt medium FM21. One liter of culture was inoculated in the fermentor until glycerol was completely consumed. Then $50 \%$ glycerol $(\mathrm{w} / \mathrm{v})$ was added at a feed rate of $18 \mathrm{ml} / \mathrm{h} / \mathrm{l}$ for $2.5 \mathrm{~h}$ until a cellular yield of $220 \mathrm{~g} / \mathrm{l}$ wet weight was achieved. When the carbon source was consumed, pure methanol was added to induce rBPTI expression. The feed rate of methanol was at $3.6 \mathrm{ml} / \mathrm{h} / 1$ for $3 \mathrm{~h}$, $7.3 \mathrm{ml} / \mathrm{h} / \mathrm{l}$ for $2 \mathrm{~h}$, and $10.9 \mathrm{ml} / \mathrm{h} / 1$ until the end of the fermentation. The fermentation lasted for $48 \mathrm{~h}$, during which, the dissolved oxygen (DO) was maintained above $20 \%$ by adjusting the rate of aeration. The $\mathrm{pH}$ was adjusted by adding $\mathrm{NH}_{4} \mathrm{OH}$ or $\mathrm{H}_{3} \mathrm{PO}_{4}$. The temperature was adjusted by adding cooled recycled water. A sample was taken to determine $\mathrm{OD}_{600}$ and wet cell weight every $6 \mathrm{~h}$, and the supernatant was used for protein analysis.

Purification of rBPTI After the fermentation, cation exchange chromatography and reverse phase chromatography were both used to purify rBPTI. After centrifugation, the supernatant was diluted 2-fold with water and the $\mathrm{pH}$ was adjusted to 4.0 with $1 \mathrm{~mol} / 1 \mathrm{NaOH}$, then $200 \mathrm{mmol} / 1$ NaAc-HAc buffer solution ( $\mathrm{pH} 4.0)$ was added to a final concentration of $20 \mathrm{mmol} / 1$. The sample was applied to a Sepharose SP XL column (GE Healthcare Bio-Sciences Corp., Piscataway, U.S.A.) pre-equilibrated with $20 \mathrm{mmol} / 1$ NaAc-HAc ( $\mathrm{pH} 4.0$ ) by gradient elution with $20 \mathrm{mmol} / 1$ $\mathrm{NaAc}-\mathrm{HAc} \quad(\mathrm{pH} \quad 4.0)$ and $2 \mathrm{~mol} / \mathrm{l} \quad \mathrm{NaCl} / 20 \mathrm{mmol} / \mathrm{l}$ $\mathrm{NaAc}-\mathrm{HAc}(\mathrm{pH} 4.0)$. The fractions with trypsin inhibition activities were pooled and 1\% trifluoroacetic acid (TFA) was added to a final concentration of $0.1 \%$. Then the sample was applied to a Source 30 RPC column (GE Healthcare Bio-Sciences Corp., Piscataway, U.S.A.) pre-equilibrated with $0.1 \%$ TFA by gradient elution with $0.1 \%$ TFA and $100 \%$ acetonitrile $/ 0.1 \%$ TFA. The fractions were analyzed with SDSPAGE to determine which contained rBPTI, and their activities were analyzed by trypsin inhibition assay. Protein concentration was determined by the Bradford method.

Mass Spectrograph and N-Terminal Amino Acid Sequence The molecular weight of rBPTI was determined by matrix-assisted laser desorption ionization-time of flight mass spectrometry (MALDI-TOF-MS-1700) at Jilin University of China. The N-terminal amino acid sequence of rBPTI was analyzed by Edman degradation method at the National Center of Biomedical Analysis (Analyzer-491, American Applied Biosystems).

Trypsin Inhibition Assay The active sites of trypsin were titrated by the method of Chase and Shaw, ${ }^{16)}$ using the burst titrant $p$-nitrophenyl guanidinobenzoate (L-BAPNA) (Sigma, St. Louis, MO, U.S.A.). This trypsin was used to titrate the trypsin inhibitory activity of rBPTI. Briefly, $10 \mathrm{~nm}$ trypsin was incubated with increasing concentrations of rBPTI in $100 \mu \mathrm{l}$ of $50 \mathrm{~mm}$ Tris- $\mathrm{HCl}, 150 \mathrm{~mm} \mathrm{NaCl}, 5 \mathrm{~mm}$ $\mathrm{CaCl}_{2}, \mathrm{pH} 7.5$ containing $0.1 \%$ bovine serum albumin in 96 well plates for $20 \mathrm{~min}$ at room temperature. The remaining trypsin activity was then measured by the change in absorbance at $405 \mathrm{~nm}$.

Therapeutic Effect of rBPTI on Acute Necrosis Pancreatitis in Rats The therapeutic effect of rBPTI on acute necrosis pancreatitis (ANP) in rats was observed in an ANP model which was induced by injection of $3.5 \%$ sodium taurocholate solution into the main pancreatic duct of rats. ${ }^{17)}$ Sixty rats were randomized into six groups (each group with 10 animals): a sham-operated control group (NS, control group), an ANP model group (ANP+NS, model group), $\mathrm{ANP}+\mathrm{rBPTI}$ group $(\mathrm{ANP}+\mathrm{rBPTI}, \mathrm{BBPT}$ group including lower dosage, medium dosage, maximum dosage) and ANP+BPTI group (ANP+BPTI, BPTI group). After establishing models, the rBPTI group received intravenous infusion of rBPTI $(40000 \mathrm{KIU} / \mathrm{kg}, 80000 \mathrm{KIU} / \mathrm{kg}, 160000 \mathrm{KIU} /$ $\mathrm{kg}$ ), the BPTI group received intravenous infusion of BPTI $(80000 \mathrm{KIU} / \mathrm{kg})$, and the control and model groups received 
intravenous infusion of physiological saline $(5 \mathrm{ml} / \mathrm{kg})$ every $8 \mathrm{~h}$ for 6 times. Forty-eight hours after the operation, the blood was collected from abdominal aorta and the serum was collected. A $10 \%$ homogenate of the pancreatic tissue was obtained. The activities of amylase (AMS) and lipase (LPS) in serum and tissue were determined. Data were presented as the mean \pm standard deviations. To analyze post hoc multiple comparisons, one-way analysis of variance and Student-Newman-Keuls test were used. Statistical analyses were performed with the SPSS statistical software package (SPSS Inc., Chicago, IL, U.S.A.). A $p$-value of $<0.05$ was considered statistically significant.

\section{RESULTS AND DISCUSSION}

Construction of Plasmid PICZC/HSA-BPTI The processing protease Kex 2 of AMF cannot cleave the protein of which the first N-terminal amino acid is alkaline amino acid. The first N-terminal amino acid of BPTI is "arginine", which is an alkaline amino acid, so the processing protease Kex2 cannot correctly cleave the pro sequence of AMF to produce natural N-terminal BPTI in P. pastoris. ${ }^{13)}$ In our study, therefore, we used HSA signal peptide to produce natural BPTI in P. pastoris.

The HSA signal peptide was subcloned to plasmid $\mathrm{PICZaC}$ to generate plasmid PICZC/HSA. The $174 \mathrm{bp}$ of gene fragment encoding the mature BPTI was amplified by RT-PCR, and subcloned into pPICZC/HSA. Thus rBPTI can be induced and secreted by HSA signal peptide and controlled by AOXI promoter (Fig. 1). Figure 1B shows the cleavage process of the prepro sequence of HSA. At first, HSA is cleaved by signal peptidase in the endoplasmic reticulum (ER) to produce proalbumin (cleavage site of P1), then the leader peptide of $\mathrm{C}$-terminal of proalbumin is cleaved by furin, a processing protease (recognition sequence is "ArgArg") in the Golgi body or secretory vesicles to produce mature albumin (cleavage site of P2). ${ }^{18)}$ Figure $1 \mathrm{C}$ shows the expected cleavage process of BPTI with HSA signal peptide. From Fig. 1B, we can find the first N-terminal amino acid of proalbumin is arginine with a cleavage site of $\mathrm{P} 1$ and the first $\mathrm{N}$-terminal amino acid of BPTI is also arginine; so the BPTI gene has been ligated with a downstream of nucleotides corresponding to HSA signal peptide, namely we speculate that this made it possible to express the correct N-terminal rBPTI. In the ER, HSA signal sequence of BPTI is cleaved by signal peptidase. However, because of the absence of recognition sequence "Arg-Arg" in the N-terminal amino acid residues of BPTI, the furin cannot cleave rBPTI in the
Golgi body or secretory vesicles, so rBPTI with the natural $\mathrm{N}$-terminal amino acid is expressed and secreted. It should be noted that we expressed rBPTI protein without C-terminal His-tag by retaining the stop codon of BPTI, which prevented the transcription of His-tagged gene. ${ }^{19)}$ Therefore, rBPTI generated in our study did not contain additional amino acids that might interfere with its biological activity.

Expression and Optimization of rBPTI Three different strains of X-33, KM71, and SMD1168 were transformed with pPICZ/HSA-BPTI, respectively. The transformants were plated on YPD plates with different concentrations of Zeocin. The number of clones was reduced with elevated concentrations of Zeocin. Forty eight of 60 clones identified by PCR were positive. In order to check the expression levels of the different strains, the 48 clones were grown and expressed in BMGY/BMMY medium, respectively; the expression results of could be easily monitored by trypsin inhibition assay (Table 1). Table 1 shows the results of the trypsin inhibition assay of 17 of the 48 positive clones. Seventeen clones with inhibition activity against trypsin of less than $0.198 \mathrm{EPU} / \mathrm{ml}$ and 14 clones without inhibition activity against trypsin are not shown in the table. Trypsin inhibition assay showed that there was a great variation between different clones in rBPTI expression. The empty vector control, of course, showed no expression of rBPTI. The activities of X-33 clones were generally higher than those of KM71 and SMD1168 clones. The activities of X-33/No. 5 and KM71/No. 22 were relatively higher, so X-33/No. 5 and

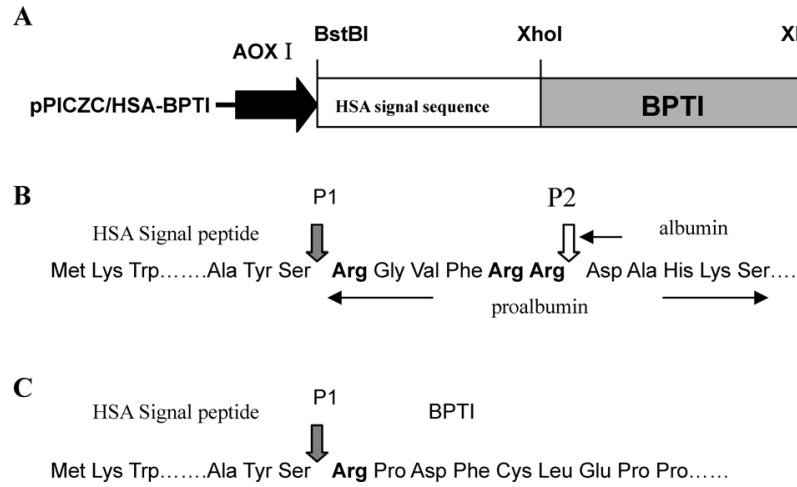

Fig. 1. Vector Construction of pPICZ/HSA-BPTI

(A) Map of the pPICZ/HSA-BPTI cloning site. (B) Cleavage of the HSA signal peptide. In the endoplasmic reticulum (ER), the proalbumin is produced by signal peptidase (P1); then in the Golgi body or secretory vesicles, the proalbumin is cleaved into mature albumin (P2). (C) Cleavage of BPTI with HSA signal peptide. In the ER, BPTI is produced and the cleavage site is $\mathrm{P} 1$; but in the Golgi body or secretory vesicles, the second cleavage does not occur because of absence of the recognition sequence of "Arg-Arg". So BPTI with the natural N-terminal amino acid is secreted.

Table 1. Analysis of the Expression of rBPTI of Different Clones by Trypsin Inhibition Assays

\begin{tabular}{|c|c|c|c|c|c|c|c|c|c|c|c|c|c|c|c|c|c|}
\hline \multirow{3}{*}{ Number of clones } & \multicolumn{17}{|c|}{ Strain } \\
\hline & \multicolumn{8}{|c|}{$X-33$} & \multicolumn{5}{|c|}{ KM71 } & \multicolumn{4}{|c|}{ SMD1168 } \\
\hline & 4 & 5 & 6 & 12 & 14 & 17 & 18 & 20 & 21 & 22 & 23 & 27 & 29 & 43 & 44 & 46 & 47 \\
\hline $\begin{array}{l}\text { Activities of rBPTI } \\
\left(\times 10^{-3} \mathrm{EPU} / \mathrm{ml}\right)\end{array}$ & 278 & 297 & 238 & 218 & 198 & 238 & 278 & 218 & 278 & 297 & 198 & 278 & 238 & 198 & 218 & 198 & 198 \\
\hline
\end{tabular}

The 48 positive colonies identified by PCR from the strains of X-33, KM71 and SMD1168 were further identified by trypsin inhibition assay. Results of the assay of 17 clones from the 48 positive clones are shown. Clones X-33/No. 5 and KM71/No. 22 were selected for further expression and identification. The first unit of aprotinin was the kallikrein inhibition unit (KIU). In recent years, the unit (KIU) of aprotinin has been changed to the European unit (EPU), and the conversion formula is as follows: EPU=1800 KIU. 
KM71/No. 22 were selected for further expression and identification. The strains X-33/No. 5 and KM71/No. 22 were incubated in $21 \mathrm{BMGY/BMMY}$ medium in a shaken flask to compare their expression levels. SDS-PAGE and trypsin inhibition assay showed that the expression level of X-33/No. 5 was higher than that of KM71/No. 22 (data not being shown), so that we selected X-33/No. 5 to further optimize its expression parameters including microbial density, temperature and $\mathrm{pH}^{20}{ }^{20}$ The strain $\mathrm{X}-33$ was a wild-type strain and may be more suitable for expression of rBPTI; it is suitable for high-density fermentation. The experiment of optimization $\mathrm{pH}$ showed that the yield of $\mathrm{rBPTI}$ was relatively higher when $\mathrm{pH}$ was 3.5 (Table 2). The $\mathrm{pH}$ of 3.5 was an acidic environment which reduced the risk of microbial contamination. The experiment of optimization temperature suggested that the microbial density at $28^{\circ} \mathrm{C}$ was close to that at $30^{\circ} \mathrm{C}$, but was twice as much as those at $24^{\circ} \mathrm{C}$ and $26^{\circ} \mathrm{C}$ (Fig. 2). A trypsin inhibition assay was also performed, and results showed that the activity of $\mathrm{rBPTI}$ at $28^{\circ} \mathrm{C}$ $(0.369 \mathrm{EPU} / \mathrm{ml})$ was higher than those at $30^{\circ} \mathrm{C}(0.357 \mathrm{EPU} /$ $\mathrm{ml}), 26{ }^{\circ} \mathrm{C}(0.264 \mathrm{EPU} / \mathrm{ml})$ and $24^{\circ} \mathrm{C}(0.231 \mathrm{EPU} / \mathrm{ml})$.

Fermentation and Purification of rBPTI After establishing the optimal parameters, rBPTI was fermented for large-scale production in an 801 fermentor. The $P$. pastoris

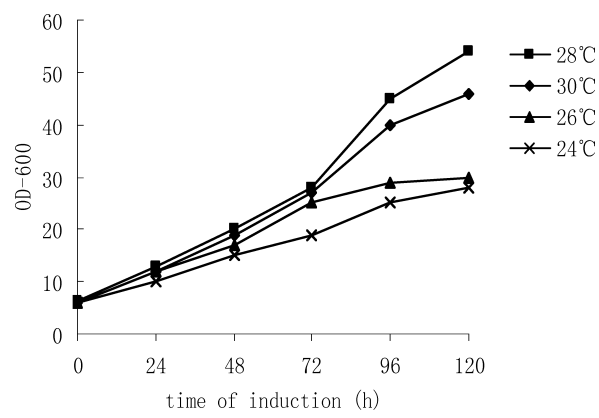

Fig. 2. Growth Curves of the P. pastoris at Different Temperature

Two liters of candidate clone was cultured in BMGY/BMMY medium at $24^{\circ} \mathrm{C}$, $26^{\circ} \mathrm{C}, 28^{\circ} \mathrm{C}$ and $30^{\circ} \mathrm{C}$ for $5 \mathrm{~d}$ and $\mathrm{OD}_{600}$ was recorded every $24 \mathrm{~h}$. The density at $28^{\circ} \mathrm{C}$ was close to that at $30^{\circ} \mathrm{C}$, and was twice as much as those at $24^{\circ} \mathrm{C}$ and $26^{\circ} \mathrm{C}$. transformant was incubated for $48 \mathrm{~h}$, which yielded $220 \mathrm{~g} / \mathrm{l}$ (yeast wet weight/broth volume). Methanol induction in a basal salt medium FM21 led to a significant expression. ${ }^{21,22)}$ The most optimal parameters were the following: FM21 culture medium containing $0.5 \%$ peptone, $\mathrm{pH} 3.5$, temperature $28^{\circ} \mathrm{C}$, yeast biomass $220 \mathrm{~g} / \mathrm{l}$, DO between $25-30 \%$ and the supply speed of methanol $8.5-9.0 \mathrm{ml} / \mathrm{h} / 1$ initial fermentation volume. The expression level of rBPTI was analyzed using SDS-PAGE, showing that the protein expression level continued to increase from 6 to $48 \mathrm{~h}$ after methanol induction, after which no further increase in level was observed. The yield of rBPTI reached $900 \mathrm{mg} / 148 \mathrm{~h}$ after fermentation. Figure 3 shows the time course of methanol-induced rBPTI expression at $28^{\circ} \mathrm{C}$ from 401 fermentation supernatant in the 801 fermentor.

Since the isoelectric point of BPTI is about 10, we first selected cation exchange chromatography to purify rBPTI. During the purification, the $\mathrm{pH}$ of buffer was selected to be 8.0, 7.0 and 6.0, respectively; however, the purification results were not ideal. When the $\mathrm{pH}$ of buffer was set to 4.0, rBPTI was separated and purified successfully. So rBPTI was first purified with Sepharose SP XL column and $\mathrm{pH}$ of the buffer was 4.0; it was eluted using $1 \mathrm{M} \mathrm{NaCl}$. Then the eluent with trypsin inhibition activities was again purified with a Source 30 RPC column and eluted with 30\% acetonitrile. After condensation, the sample showed a single band with a molecular weight of $6.5 \mathrm{kDa}$ accounting for over $95-98 \%$ of the total protein in $15 \%$ SDS-PAGE. The purity was more than $95 \%$ and the purified coefficient was over $60 \%$ (Table $3)$.

Mass Spectrograph and N-Terminal Amino Acid Sequence The molecular weight of the purified rBPTI was 6508 Da measured with a mass spectrograph and the theoretical value of molecular weight of BPTI was 6511. Sequence of N-terminal 15 amino acids of rBPTI was measured at the National Center of Biomedical Analysis and was consistent with the natural BPTI.

Trypsin Inhibition Assay BPTI is a member of the $\mathrm{Ku}-$ nitz-type inhibitor family and possesses the ability to inhibit

Table 2. Analysis of the Activities of rBPTI in Media of Different pH by Trypsin Inhibition Assays

\begin{tabular}{cccccccccc}
\hline \hline $\mathrm{pH}$ value & 3.0 & 3.5 & 4.0 & 4.5 & 5.0 & 5.5 & 6.0 & 6.5 & 7.0 \\
\hline rBPTI activity $\left(\times 10^{-3} \mathrm{EPU} / \mathrm{ml}\right)$ & 297 & 317 & 297 & 297 & 238 & 238 & 238 & 218 & 198 \\
\hline
\end{tabular}

The selected colony was incubated in $10 \mathrm{ml}$ BMGY/BMMY media of different $\mathrm{pH}$. The highest expression level of rBPTI was observed in medium of pH 3.5 .

A

$\begin{array}{lllllllll}1 & 2 & 3 & 4 & 5 & 6 & 7 & 8 & 9\end{array}$

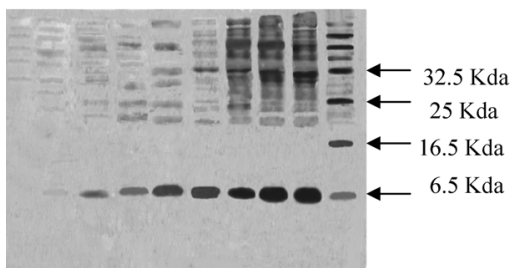

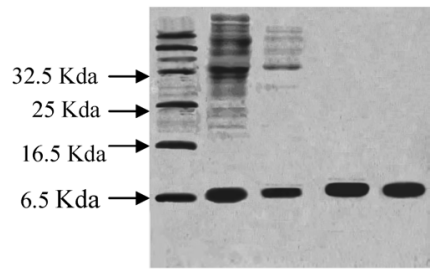

Fig. 3. Analysis of rBPTI with $15 \%$ SDS-PAGE

Supernatants were collected at the indicated times after methanol induction. Proteins were separated on 15\% SDS-PAGE and stained with Coomassie brilliant blue. (A) Time course of methanol-induced expression of rBPTI. Lanes 1-9: fermentation supernatant at $0 \mathrm{~h}, 6 \mathrm{~h}, 12 \mathrm{~h}, 8 \mathrm{~h}, 24 \mathrm{~h}, 30 \mathrm{~h}, 36 \mathrm{~h}, 42 \mathrm{~h}$ and $48 \mathrm{~h}$, respectively; lane 10 : protein molecular size marker. (B) Analysis of purification. Lane 1: Protein molecular size marker; lane 2: Culture supernatant at 48 h; lane 3: Eluent from Sepharose SP XL; lane 4: Eluent from Source 30 RPC; lane 5: Standard product of BPTI. 
Table 3. Purification of rBPTI from 401 Culture Supernatant of P. pastoris

\begin{tabular}{lccccc}
\hline \hline \multicolumn{1}{c}{ Step } & Total protein $(\mathrm{mg})$ & Total activity (EPU) & Specific activity (EPU/mg) & Total yield (\%) & Purification multiple \\
\hline Culture supernatant & $4.4 \times 10^{4}$ & $3.176 \times 10^{4}$ & 0.72 & 100 & 70 \\
Sepharose SP XL & $8 \times 10^{3}$ & $2.222 \times 10^{4}$ & 2.78 & 62 & 5.5 \\
Source 30 RPC & $6 \times 10^{3}$ & $1.985 \times 10^{4}$ & 3.30 & 6.3 \\
\hline
\end{tabular}

Data are from 401 of $P$. pastoris culture supernatant $48 \mathrm{~h}$ after methanol induction.

Table 4. Effect of rBPTI on the Activities of AMS and LPS in Serum and Tissue in ANP Model of Rats

\begin{tabular}{|c|c|c|c|c|c|}
\hline Group & Dose (KIU/kg) & AMS (U/gprot) (in serum) & LPS (U/gprot) (in serum) & AMS (U/gprot) (in tissue) & LPS (U/gprot) (in tissue) \\
\hline Control & - & $2284 \pm 304$ & $0.426 \pm 0.053$ & $16156 \pm 2044$ & $221 \pm 27$ \\
\hline Model & - & $5439 \pm 670^{\# \#}$ & $1.957 \pm 0.556^{\# \#}$ & $36917 \pm 6908^{\# \#}$ & $545 \pm 44^{\# \#}$ \\
\hline \multirow[t]{3}{*}{ rBPTI } & $4 \times 10^{4}$ & $3013 \pm 480 * *$ & $1.234 \pm 0.341^{*}$ & $28467 \pm 2407 *$ & $291 \pm 23 * *$ \\
\hline & $8 \times 10^{4}$ & $2830 \pm 540 * *$ & $1.115 \pm 0.450^{*}$ & $28527 \pm 2312 *$ & $282 \pm 35^{* *}$ \\
\hline & $16 \times 10^{4}$ & $2765 \pm 460 * *$ & $1.108 \pm 0.204^{*}$ & $24343 \pm 1723 * *$ & $292 \pm 46^{* *}$ \\
\hline BPTI & $8 \times 10^{4}$ & $2936 \pm 340 * *$ & $1.113 \pm 0.416^{*}$ & $25791 \pm 2819 * *$ & $290 \pm 42 * *$ \\
\hline
\end{tabular}

\# $p<0.01$ vs. control; $* p<0.05, * * p<0.01 v s$. model. Results showed that the rat ANP model was established successfully. Comparison of the rBPTI group with the model group showed the activities of AMS and LPS in serum and tissue were significantly decreased $(p<0.05, p<0.01)$. U/gprot is a measuring unit of enzyme.

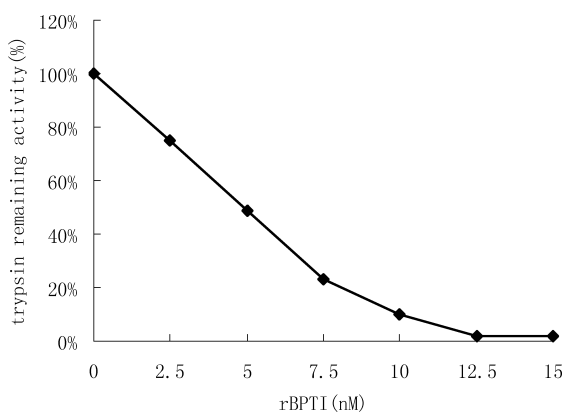

Fig. 4. Titration of the Protease Inhibition Activity of Purified rBPTI with Trypsin

Ten nanomolar trypsin was incubated with increasing concentrations of purified rBPTI and the residual trypsin activity was measured using the chromogenic substrate. Titration experiments revealed a one-to-one stoichiometry for trypsin inhibition.

trypsin. So trypsin inhibition assays were performed to analyze the activity of the purified rBPTI and the difference compared with the natural BPTI. Trypsin inhibition assays of the purified rBPTI indicated one-to-one stoichiometry for trypsin inhibition (Fig. 4) and inhibition constant $\left(K_{\mathrm{j}}\right)$ was $2.6 \pm 0.1 \times 10^{-9}$, which was in accord with that of natural BPTI. This demonstrated that $\mathrm{rBPTI}$ expressed in P. pastoris folded correctly and possessed the same bioactivity of trypsin inhibition as BPTI.

Therapeutic Effect of rBPTI on Acute Necrosis Pancreatitis in Rats BPTI (aprotinin) had been used in the treatment of acute pancreatitis, so the therapeutic effect of rBPTI was first observed in the rat ANP model. Compared with the control group, the activities of AMS and LPS in serum and tissue in model group were significantly increased $(p<0.01)$, indicating that the rat ANP model had been established successfully. In the rBPTI group the activities of AMS and LPS in serum and tissue were significantly decreased compared with the model group $(p<0.05, p<0.01)$ (Table 4$)$. This suggested that $\mathrm{rBPTI}$ inhibited the high secretion condition of ANP to relieve pancreatic auto-digestion and had a therapeutic effect on ANP in rats as did BPTI.

\section{CONCLUSION}

In summary, we have described a method of preparing rBPTI which possesses a natural N-terminal amino acid sequence (Arg-Pro-Asp) with signal peptide from HSA and a recombinant DNA technique in $P$. pastoris. rBPTI was expressed as a heterologous preprotein having the signal peptide from HSA at its N-terminal, and this preprotein was recognized and correctly processed by P. pastoris signal peptidase. Through the optimization of fermentation parameters, the concentration of rBPTI in an 801 fermentor reached $900 \mathrm{mg} / \mathrm{l}$. A simple purification method was established which led to a pure protein, structurally and functionally similar to the natural BPTI. The therapeutic effect of rBPTI to ANP in rats was also identified in an ANP rat model.

The purity of the rBPTI did not reach as high as expected. However, it is possible that the purity may be further enhanced by optimizing purification conditions and choosing more suitable resins. Therefore, the yeast system described here provides a strong foundation for the further development of P. pastoris-produced rBPTI and its future clinical application.

Acknowledgements This work was supported by grants from the National High Technology Research and Development Program of China (No. 2004AA205020). We thank Dr. Yan Fang (Vanderbilt University) for suggestions for the manuscript preparation.

\section{REFERENCES}

1) Hagihara Y., Shiraki K., Nakamura T., Uegaki K., Takagi M., Imanaka T., Yumoto N., J. Biol. Chem., 277, 51043-51048 (2002).

2) Barbar E., Hare M., Makokha M., Barany G., Woodward C., Biochemistry, 40, 9734-9742 (2001).

3) Antuch W., Berndt K. D., Chávez M. A., Delfín J., Wüthrich K., Eur. J. Biochem., 212, 675-684 (1993).

4) Pritchar L., Dufton M. J., J. Mol. Biol., 285, 1589-1607 (1999).

5) Bull D. A., Connors R. C., Albanil A., Reid B. B., Neumayer L. A., Nelson R., Stringham J. C., Karwande S. V., J. Thorac. Cardiovasc. Surg., 119, 242-250 (2000).

6) Molenaar I. Q., Begliomini B., Grazi G. L., Ringers J., Terpstra O. T., 
Porte R. J., Transplantation, 71, 247-252 (2001).

7) Marks C. B., Vasser M., Ng P., Henzel W., Anderson S., J. Biol. Chem., 261, 7115-7118 (1986).

8) Nilsson B., Berman-Marks C., Kuntz I. D., Anderson S., J. Biol. Chem., 266, 2970-2977 (1991).

9) Chesshyre J. A., Kraunsoe J. A., Lowe G., Biotechnol. Appl. Biochem., 22, 269-280 (1995).

10) Bannister S. J., Wittrup K. D., Biotechnol. Bioeng., 68, 389-395 (2000).

11) Rakestraw A., Wittrup K. D., Biotechnol. Bioeng., 65, 374-378 (2005).

12) Auerswald E. A., Schroder W., Kotick M., Biol. Chem. Hoppe. Seyler, 368, 1413-1425 (1987).

13) Vedvick T., Buckholz R. G., Engel M., Urcan M., Kinney J., Provow S., Siegel R. S., Thill G. P., J. Ind. Microbiol., 7, 197-201 (1991).

14) MacKenzie D. A., Kraunsoe J. A., Chesshyre J. A., Lowe G., Komiyama T., Fuller R. S., Archer D. B., J. Biotechnol., 63, 137-146
(1998).

15) Huaping L., Jie L., Zhifeng W., Yingchang Z., Yuhuan L., Biol. Pharm. Bull., 30, 1851-1855 (2007).

16) Chase T. Jr., Shaw E., Biochem. Biophys. Res. Commun., 29, 508-514 (1967).

17) Oruc N., Ozutemiz A. O., Yukselen V., Nart D., Celik H. A., Yuce G., Batur Y., Pancreas, 28, $1-8$ (2004).

18) Brennan S. O., Peach R. J., J. Biol. Chem., 266, 21504-21508 (1991).

19) Cregg J. M., Madden K. R., Barringer K. J., Thill G. P., Stillman C. A., Mol. Cell. Biol., 9, 1316-1323 (1989).

20) Sreekrishna K., Brankamp R. G., Kropp K. E., Blankenship D. T., Tsay J. T., Smith P. L., Wierschke J. D., Subramaniam A., Birkenberger L. A., Gene, 190, 55-62 (1997).

21) Cregg J. M., Vedvick T. S., Raschke W. C., Biotechnology, 11, 905910 (1993).

22) Cregg J. M., Cereghino J. L., Shi J., Higgins D. R., Mol. Biotechnol., 16, 23-52 (2000). 